\title{
'Take-away' foraging spatially uncouples predator and prey-attack distributions
}

\author{
Isabel M. Smallegange ${ }^{1 *} \dagger$, Jaap van der Meer ${ }^{1,2}$ and Maurice W. Sabelis ${ }^{3}$ \\ ${ }^{1}$ Royal Netherlands Institute for Sea Research (NIOZ), Department of Marine Ecology and Evolution, P.O. Box 59, 1790 AB, \\ Den Burg (Texel), The Netherlands; ${ }^{2}$ Free University, Institute of Ecological Science, De Boelelaan 1085, 1081 HV \\ Amsterdam, The Netherlands; and ${ }^{3}$ Institute for Biodiversity and Ecosystem Dynamics, Section Population Biology, \\ P.O. Box 94084, 1090 GB, Amsterdam, The Netherlands
}

\begin{abstract}
Summary
1. Ideal-free distribution theory assumes that in a patchy environment foragers maximize fitness and hence their feeding rate by balancing gains from more food against losses from more competition. Ultimately, individuals cannot increase their feeding rate by moving to another patch and they distribute themselves over patches in proportion to prey density per patch.

2. In our experiments with shore crabs Carcinus maenas foraging on two adjacent patches with mussels Mytilus edulis, the implicit assumption of ideal-free distribution theory that catch should match time spent in a prey patch is not met, however. Despite aggregating their attack where it is most profitable shore crabs distributed themselves homogeneously across mussel patches: they 'take away' the prey caught and consume it elsewhere to reduce interference.

3. Thus, predator distributions can be quite different from prey-attack distributions. This is important because the latter is shown to be decisive for persistence of predator and prey populations in ecological models.
\end{abstract}

Key-words: foraging behaviour, interference competition, population stability, predator-prey dynamics, travelling costs

\section{Introduction}

Classic predator-prey theory is based on the assumption of homogeneous mixing in populations (Lotka 1925; Volterra 1926; Nicholson \& Bailey 1935; Rosenzweig \& MacArthur 1963). However, in nature predators tend to aggregate in patches harbouring more prey (Stiling 1987; Walde \& Murdoch 1988) and precisely this feature has been shown to matter to the stability of predator-prey models, based on phenomenological distributions (Hassell \& May 1973; Chesson \& Murdoch 1986; Murdoch \& Stewart-Oaten 1989), or by ideal-free distributions (IFDs) (Van Baalen \& Sabelis 1993, 1999; Bernstein, Auger \& Poggiale 1999; Hassell 2000; Ranta, Lundberg \& Kaitala 2006).

In IFD theory, predators are assumed to have equal competitive ability, perfect knowledge on habitat qualities, and free choice to move to the habitat that maximizes their feeding rate. In deciding where to forage, predators balance the gains from more prey per patch against the loss from increased competition. This results in distributions where no

*Correspondence author. E-mail: i.smallegange@imperial.ac.uk $\dagger$ Present address: Division of Biology, Imperial College London, Silwood Park, Ascot, SL5 7PY, UK. individual can increase its feeding rate by moving to another patch and this outcome is termed the ideal-free distribution (Fretwell \& Lucas 1969). Ideal-free theory, however, is also based on an assumption that is rarely made explicit: the actual predator distribution reflects the distribution of attacks on prey. Yet, in absence of between-patch travelling costs, predators could reduce interference from competitors by handling a prey item on an alternative patch with fewer competitors.

Thus, the actual distribution of predators may not reflect the distribution of attacks on prey, contrary to what has been assumed in the vast majority of empirical studies (Stiling 1987; Milinski \& Parker 1991; Tregenza 1995). This may have far-reaching consequences for the predictions of predator-prey models. For example, if predators are observed to be distributed equally across food patches, even though they only attack prey on the better food patches, the predicted predator-prey dynamics are likely to be unstable if the observed distribution of predators is taken as their preyattack distribution (Hassell \& May 1973). However, the actual, aggregated prey-attack distribution likely results in stable predator-prey dynamics (May 1978; Van Baalen \& Sabelis 1993; Bernstein, Auger \& Poggiale 1999). It is therefore important to perform empirical tests of IFD theory and 
its underlying assumptions. Here, we present the results of such tests using shore crabs Carcinus maenas L. that forage on a standing stock of mussels Mytilus edulis L. Shore crabs are known to avoid conspecifics while handling prey (Smallegange \& van der Meer 2007) and may therefore be prone to move between patches under ideal-free conditions. In two previous studies (Smallegange, van der Meer \& Kurvers 2006; Smallegange \& van der Meer 2007) we assessed feeding rates for a single crab that foraged alone or with an equal competitor in a single patch. From these previous studies we derived ideal-free predictions on food uptake and patch choice for a focal crab foraging with an equal competitor. We tested this ideal-free hypothesis against the null hypothesis that crabs have no preference, in a closed 'habitat' where crabs foraged on two adjacent patches that differed in prey density. To prevent prey depletion during these experiments we used a novel experimental set-up (Smallegange, van der Meer \& Kurvers 2006) that allows for quick replenishment of the prey consumed.

\section{Materials and methods}

\section{GENERAL OUTLINE}

In this paper we discuss four experiments: Exp. 1, Exp. 2, Exp. 3a, and Exp. 3b. Exp. 1 and 2 are published experiments in which we assessed the feeding rates of crabs foraging on a single food patch (Exp. 1: Smallegange, van der Meer \& Kurvers 2006; Exp. 2: Smallegange $\&$ van der Meer 2007). The patch choice experiments $3 \mathrm{a}$ and $3 \mathrm{~b}$ were conducted for the study reported here. In all experiments we used male shore crabs of the same size; i.e. their major claw length (MCL) was between 29 and $35 \mathrm{~mm}$ (corresponding to 51-58 mm carapace width). Laboratory conditions in all experiments were standardized: the temperature of the seawater was $15-21{ }^{\circ} \mathrm{C}$ and the photoperiod was kept at a $12 \mathrm{~h}$ light: $12 \mathrm{~h}$ dark cycle with experiments carried out in the light period. In all experiments, prey were offered at three different densities: 4, 16, and 32 mussels (M. edulis) per food patch (one food patch equalled $1 / 4 \mathrm{~m}^{2}$ ).

Exp. $3 \mathrm{a}$ and $3 \mathrm{~b}$ were designed to test two hypotheses on patch choice of two shore crabs foraging on two patches (the experimental set-up is explained below). The null hypothesis states that crabs have no preference for a food patch, and the ideal-free hypothesis states that crabs choose the food patch where they can maximize their food uptake while minimizing costs of competition. We quantified each hypothesis using 1- and 2-predator, continuous-time Markov chains that describe the foraging process of a predator foraging alone or with one competitor on a single food patch (van der Meer \& Smallegange 2009). We used trials from Exp. 1 and 2 to estimate the parameters of the 1- and 2-predator Markov chains (see below). We then used the Markov chains to quantify the null hypothesis and ideal-free hypothesis on patch choice, which we subsequently tested against the results obtained in patch choice experiments $3 \mathrm{a}$ and $3 \mathrm{~b}$.

\section{COLLECTION AND MAINTENANCE OF ANIMALS}

Male shore crabs that were used in experiments $3 \mathrm{a}$ and $3 \mathrm{~b}$ were caught in July 2003 (Exp. 3a) and July 2004 (Exp. 3b) from a fyke (a long, bag-shaped fishing net held open by hoops) located at the south-tip of Texel, The Netherlands, and were maintained in individual holding tanks $(22 \times 14 \times 13 \mathrm{~cm})$ that were submerged in a large basin with continuously running seawater. Temperature in the basin was $15^{\circ} \mathrm{C}$. All crabs were of the same size as in Exp. 1 and 2: 29$35 \mathrm{~mm}$ MCL. Crabs were allowed at least 1 week to acclimatize to laboratory conditions. Intertidal mussels were scraped from ballast piers at the coast of Den Helder, the Netherlands in June 2003 (Exp. 3a) and June/July 2004 (Exp. 3b), cleaned of any attached, fouling organisms, and maintained in a separate large basin with continuously running seawater. In the experiments we used mussels of maximum length 12-14 mm (as in Exp. 1 and 2).

\section{EXPERIMENTAL SET-UP}

To prevent prey depletion and hence effects of exploitation competition while conducting experiments $3 \mathrm{a}$ and $3 \mathrm{~b}$, we used an experimental tank specifically designed to replenish consumed prey without disturbing the crabs. The tank contained two adjacent food patches (Fig. 1). Both food patches rotated slowly at a constant speed $\left(10^{-2} \mathrm{rad}\right.$ per s); slow enough to prevent disturbing the behaviour of the crabs. The patches were separated by a partition (Fig. 1), creating a visual barrier. Crabs could walk between patches through three openings in the partition. The size of each food patch equalled that of the food patches used in Exp. 1 and 2 (i.e. $1 / 4 \mathrm{~m}^{2}$ ).

Each trial started by introducing two crabs into the tank: one on each (randomly assigned) patch. One crab was the focal crab, the other the competitor crab. We analysed the behaviour of the focal crab to obtain independent observations. Focal and competitor crabs were randomly selected from a stock of 40 crabs (Exp. 3a) and 16 crabs (Exp. 3b). Crabs were allowed to acclimatise to the tank for $30 \mathrm{~min}$, after which trials started. Trials lasted $45 \mathrm{~min}$. During the acclimatization period and following trial period, crabs foraged on mussels. However, the total time period that crabs were in the tank was not long enough for crabs to reach satiation levels (Smallegange, unpublished data). Trials were videotaped using a Sony digital video camera recorder DCR-TRV900E (Sony, Berlin, Germany). We used the Observer 3.0 (Noldus Information Technology, Wageningen, the Netherlands) as an event recorder, which allowed us to continuously monitor on which patch each crab was foraging. We scored the time spent handling prey by the focal crab, and the total number of mussels that the focal crab consumed. We also scored the number of times that the focal crab walked from one patch to the other, and the total number of mussels that the focal crab consumed on each patch. In Exp. 1 and 2, per capita feeding rates were measured at mussel densities 4,16 , and 32 per food patch. Therefore, in the patch choice

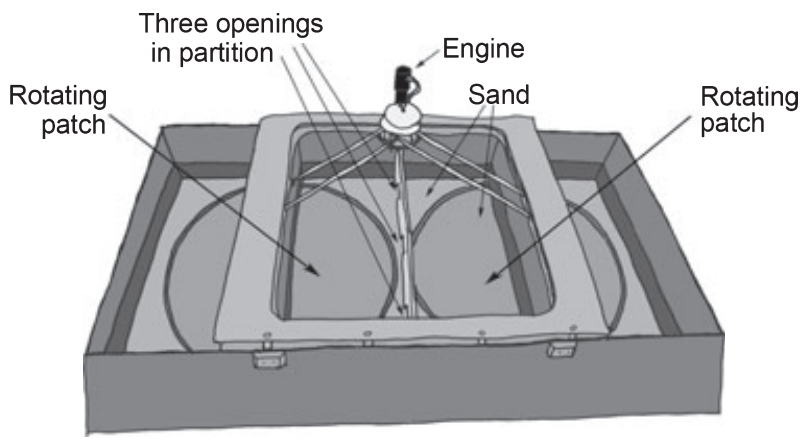

Fig. 1. Scheme of experimental tank showing both food patches that rotated during trials and the partition that separated both food patches. Crabs could walk between food patches through three openings in the partition. The observer could replenish any eaten mussel in that part of a food patch that was not accessible to the crabs (i.e. outer left and outer right part of tank). 
experiments we adhered to these densities and the number of mussels on one patch was always 4 and on the other patch 4,16 or 32 . This created three treatments, or patch combinations: '4-4', '4-16', and '432'. Treatments were replicated four times (Exp. 3a) and twice (Exp. 3b). Mussel densities were randomly assigned to each of the two patches.

\section{STATISTICAL ANALYSIS OF EXPERIMENTAL RESULTS}

We explored four response variables: (1) the food intake of a focal crab on the rich patch relative to that on the poor patch, (2) the fraction of time that a focal crab spent on the rich patch, (3) the handling time per mussel capture on the rich patch relative to that on the poor patch and (4) the number of times that the focal crab moved, with its prey, away from its competitor on the rich patch to the unoccupied poor patch, minus the number of times that the focal crab moved, with its prey, away from its competitor on the poor patch to the unoccupied, rich patch. Time fractions were arcsine-transformed and ratios were log-transformed to approach normality. Zero values $(n=2)$ for observed food intakes were replaced with the lowest observed value. We calculated bootstrap confidence intervals (CIs) around the mean of each response variable by taking a thousand bootstrap samples (in MatLab 6.5) from the response values observed for each patch combination. To prevent type I errors, a Bonferroni correction was applied and the level of significance was set at $\alpha=0.05 / n=0.017$, where $n$ equals the number of treatments ( 3 in this case). This means that any values falling outside of these CIs are significant at the $5 \%$ level. Response variables (1) and (2) were used to test the null and ideal-free hypothesis by comparing these 95\% CIs against the predictions under the null and ideal-free hypotheses (which were quantified using 1- and 2-predator Markov chains: see below). Response variable (3) was used to assess if prey-handling times differed depending on whether the focal crab was on the rich or poor patch, by assessing if the $95 \%$ CI differed from the ratio of unity (which equals no difference). Response variable (4) was used to assess if the foraging behaviour of crabs that were handling prey in the presence of the competitor differed depending on whether they were on the rich or on the poor patch, by assessing if the $95 \%$ CIs differed from zero (which equals no difference). In one trial (Exp. 3a: patch combination '4-16') the focal crab did not eat at all, and this trial was discarded from all analyses as the focal crab did not display any foraging behaviour. It turned out that the results of the two experiments did not differ qualitatively, and since we had no a priori expectation that crabs or mussels would behave differently in the different years in which the experiments were performed, we pooled the data of both experiments to increase statistical power.

\section{1- AND 2-PREDATOR MARKOV CHAINS}

The continuous-time Markov chains reflect the behaviour of shore crabs foraging on mussels, and describe the foraging process of a predator foraging alone or with one competitor on a single food patch (van der Meer \& Smallegange 2009). In the Markov chains, a predator can be in one of four behavioural states: searching, $\mathrm{S}$, handling, $\mathrm{H}$, fighting after searching with a searcher, $\mathrm{F}$, and fighting after searching or handling with a handler or searcher respectively, G. The states of each Markov chain give all possible combinations of behaviours that predators may display at one time (Table 1). A transition between states entails that one individual changes its behaviour from searching to handling or vice versa, or that two individuals start or stop fighting. In the 1-predator Markov chain, the transition rate from, for example, the state $\mathrm{S}$ (the predator is searching) to $\mathrm{H}$ (the
Table 1. Transition matrix of the 1-predator and 2-predator Markov chain. Letters denote the different states of each chain, which give all possible combinations of behaviours that predators may display at one time: searching $(\mathrm{S})$, handling $(\mathrm{H})$, fighting with a searcher after searching $(\mathrm{F})$, or fighting with a searcher or handler after handling or searching respectively $(\mathrm{G}) . v$ is the search area for prey per unit time, $D$ the density of prey, $\mu$ the search area for competitors per unit time, $\lambda$ the inverse of the handling time and $\varphi$ the inverse of the duration of an interaction

\begin{tabular}{|c|c|c|c|c|c|c|c|c|c|}
\hline & \multicolumn{2}{|c|}{$\begin{array}{l}\text { One } \\
\text { predator }\end{array}$} & & \multicolumn{6}{|c|}{ Two predators } \\
\hline & $\mathbf{S}$ & $\mathrm{H}$ & & SS & $\mathrm{SH}$ & HS & $\mathrm{HH}$ & FF & GG \\
\hline S & - & $v D$ & SS & - & $v D$ & $v D$ & 0 & $\mu$ & 0 \\
\hline \multirow[t]{5}{*}{$\mathrm{H}$} & $\lambda$ & - & $\mathrm{SH}$ & $\lambda$ & - & 0 & $v D$ & 0 & $\mu$ \\
\hline & & & HS & $\lambda$ & 0 & - & $v D$ & 0 & $\mu$ \\
\hline & & & $\mathrm{HH}$ & 0 & $\lambda$ & $\lambda$ & - & 0 & 0 \\
\hline & & & $\mathrm{FF}$ & $\varphi$ & 0 & 0 & 0 & - & 0 \\
\hline & & & GG & 0 & $\varphi$ & $\varphi$ & 0 & 0 & - \\
\hline
\end{tabular}

predator is handling) is the prey capture rate and equals $v D$ (Table 1), where $D$ is the prey density, and $v$ the area searched for prey per unit time. Similarly, in the 2-predator Markov chain, the transition rate from SS (both predators are searching) to HS (the focal predator is handling, the other is still searching) equals $v D$ (Table 1). The transition rate from $\mathrm{H}$ to $\mathrm{S}$ (and also from, for example, HS to SS or $\mathrm{HH}$ to $\mathrm{SH}$ ) equals $\lambda$, and the expected handling time of a prey item is thus $1 / \lambda$ (s) (Table 1). The transition rate from a state where two predators are foraging to a state where they have started a fight equals $\mu$, which is the area searched for predators per unit time (Table 1). Two searching predators that start a fight is a transition from the state SS to FF (Table 1). A handling predator and a searching predator that start a fight is a transition from the state $\mathrm{SH}$ or HS to GG (Table 1). Two handling predators do not engage in fights. The transition rate of going from fighting back to foraging, for example, from the state FF back to SS, is $\varphi$ (Table 1), which is the rate at which fights come to an end. The expected duration of a fight is therefore $1 / \varphi(s)$. Using the results of Exp. 1 and 2 we estimated the parameters using a maximum likelihood approach (van der Meer \& Smallegange 2009) at: $\lambda: 0 \cdot 01 \mathrm{~s}^{-1}, \varphi: 0.09 \mathrm{~s}^{-1}, v: 3 \cdot 15$ $\mathrm{cm}^{2} \mathrm{~s}^{-1}$ (Holling) and $2.63 \mathrm{~cm}^{2} \mathrm{~s}^{-1}$ (Beddington), and $\mu$ : 42.12 $\mathrm{cm}^{2} \mathrm{~s}^{-1}$.

\section{DERIVING PREDICTIONS ON PATCH CHOICE: NULL HYPOTHESIS}

Under the null hypothesis we expect crabs to have no preference, thereby giving rise to constant dispersal rates between patches while searching for prey. In that case, crabs should spend fifty percent of their searching time on either patch. However, shore crabs have relatively long handling times (Smallegange \& van der Meer 2003), and are thus expected (even when they have no preference for the more profitable patch) to spend more than fifty percent of their foraging time (which includes handling time) on the more profitable patch. To calculate expected values under the null hypothesis for the first two response variables, we therefore modified the 2-predator Markov chain such that each predator could be alone on a patch or both could be on the same patch. Again, each predator is either searching $(\mathrm{S})$, handling $(\mathrm{H})$, fighting after searching with a searcher $(\mathrm{F})$, and 
fighting after searching or handling with a handler or searcher, respectively $(G)$ and the chain therefore consists of twenty states: $\mathrm{S}_{1} \mathrm{~S}_{1}, \mathrm{~S}_{1} \mathrm{H}_{1}, \mathrm{H}_{1} \mathrm{~S}_{1}, \mathrm{H}_{1} \mathrm{H}_{1}, \mathrm{~F}_{1} \mathrm{~F}_{1}, \mathrm{G}_{1} \mathrm{G}_{1}, \mathrm{~S}_{2} \mathrm{~S}_{1}, \mathrm{~S}_{2} \mathrm{H}_{1}, \mathrm{H}_{2} \mathrm{~S}_{1}, \mathrm{H}_{2} \mathrm{H}_{1}, \mathrm{~S}_{1} \mathrm{~S}_{2}$, $\mathrm{S}_{1} \mathrm{H}_{2}, \mathrm{H}_{1} \mathrm{~S}_{2}, \mathrm{H}_{1} \mathrm{H}_{2}, \mathrm{~S}_{2} \mathrm{~S}_{2}, \mathrm{~S}_{2} \mathrm{H}_{2}, \mathrm{H}_{2} \mathrm{~S}_{2}, \mathrm{H}_{2} \mathrm{H}_{2}, \mathrm{~F}_{2} \mathrm{~F}_{2}$, and $\mathrm{G}_{2} \mathrm{G}_{2}$, where numbers denote patch 1 or patch 2, and the two letters of each state denote which behaviour each predator displays (Fig. 2). Transition probabilities are as in van der Meer \& Smallegange (2009), with the addition that searchers move between patches with the rate $\theta$, for example, the transition rate from $S_{1} S_{1}$ to $S_{2} S_{1}$ equals $\theta$ (Fig. 2).

The probability that a continuous-time Markov chain will be in state $\mathrm{j}$ at time $\mathrm{t}$ (generally) converges to a limiting value, or limiting probability, independent of the initial state (Ross 1989). Simulations showed that this condition holds for the 1- and 2-predator Markov chains (Smallegange \& van der Meer 2010) as well as for the 2-patch2-predator Markov chain (Supporting Information). Since the Markov chain represents a foraging process, the limiting probability of each state is the fraction of time that the foraging process is in that state. In this current study, we express the limiting probabilities relative to the all-predators-are-searching state, and hence we refer to these limiting probabilities as relative, limiting probabilities. The relative, limiting probabilities are calculated from the set of associated balance equations of the Markov chain. These balance equations state that in the long run the rate at which the process enters state $\mathrm{j}$ equals the rate at which the process leaves state $\mathrm{j}$. For two predators foraging on two food patches (Fig. 2), the balance equations are given in Table 2. The expected proportion of time that a predator searches on a patch $\left(\mathrm{S}^{*}\right)$ can be calculated from the relative, limiting probabilities (Table 2). For example, the search time of the first crab on patch 1 is the sum of the relative, limiting probabilities of states 1 , 2,11 and 12 which, divided over the limiting probabilities of states $1-$ 6 and 11-14, gives the proportion of time spent searching on patch 1 by the first crab (Table 2). Multiplying this proportion with $v \mathrm{D}$ on each patch gives the expected per capita feeding rate on each patch. The null hypothesis for the first response variable is thus given by:

$\frac{W_{r}}{W_{p}}=\frac{v D_{r} S_{r}^{*}}{v D_{p} S_{p}^{*}}$

where subscripts denote either the rich patch (r) or the poor patch (p), and D is the prey density on a patch. From the relative limiting probabilities we also calculated the proportion of time that a predator is expected to stay on the rich patch under the null hypothesis (second response variable). For example, the total time that the first crab is on patch 1 is the sum of the limiting probabilities of states $1-6$ and 11-14 (Table 2), which, divided over the sum of the limiting probabilities of all states, gives the fraction of time that the first crab is expected to forage on patch 1 .

\section{DERIVING PREDICTIONS ON PATCH CHOICE: IDEAL- FREE HYPOTHESIS}

We modelled expected per capita feeding rates by deriving the expected proportion of time that a predator searches (alone or in the presence of a competitor) from the 1- and 2-predator Markov chains (Supporting Information), and multiplying this proportion by $v D$. For a predator foraging alone, this results in (Supporting Information):

$W=\frac{v D}{1+\frac{v D}{\lambda}}$.

This equation is the well-known functional response of Holling (Holling 1959). For a predator foraging with one equal competitor, the expected per capita feeding rate equals (van der Meer \& Smallegange 2009):

$$
W=\frac{v D}{1+\frac{v D}{\lambda}+\frac{\mu}{\varphi} \frac{1+2 v D / \lambda}{1+v D / \lambda}} .
$$

This equation is the one-competitor analogue of Beddington's functional response (Beddington 1975). Beddington's functional response is a well-known extension of Holling's functional response to include competition between predators.

Incorporating the above given parameter estimates for $v, \mu, \lambda$, and $\varphi$ (here expressed per $1 / 4 \mathrm{~m}^{2}$ by multiplying the estimates for $v$ and $\mu$ by $4 / 10000$, and with $\lambda$ and $\varphi$ expressed $\mathrm{s}^{-1}$ ) revealed that the predicted per capita feeding rates for mussel densities 4,16 , and 32 (\# per 1/4 $\mathrm{m}^{2}$ ) were (Holling) 0·20, 0.40, and 0.48, and (Beddington) $0 \cdot 15,0 \cdot 34$, and 0.43 prey per min (estimated feeding rates were in $\# \mathrm{~s}^{-1}$ and therefore multiplied by 60 to obtain feeding rates in \# $\min ^{-1}$ ). To derive ideal-free predictions for the second response variable we compared these predicted feeding rates. For example, for

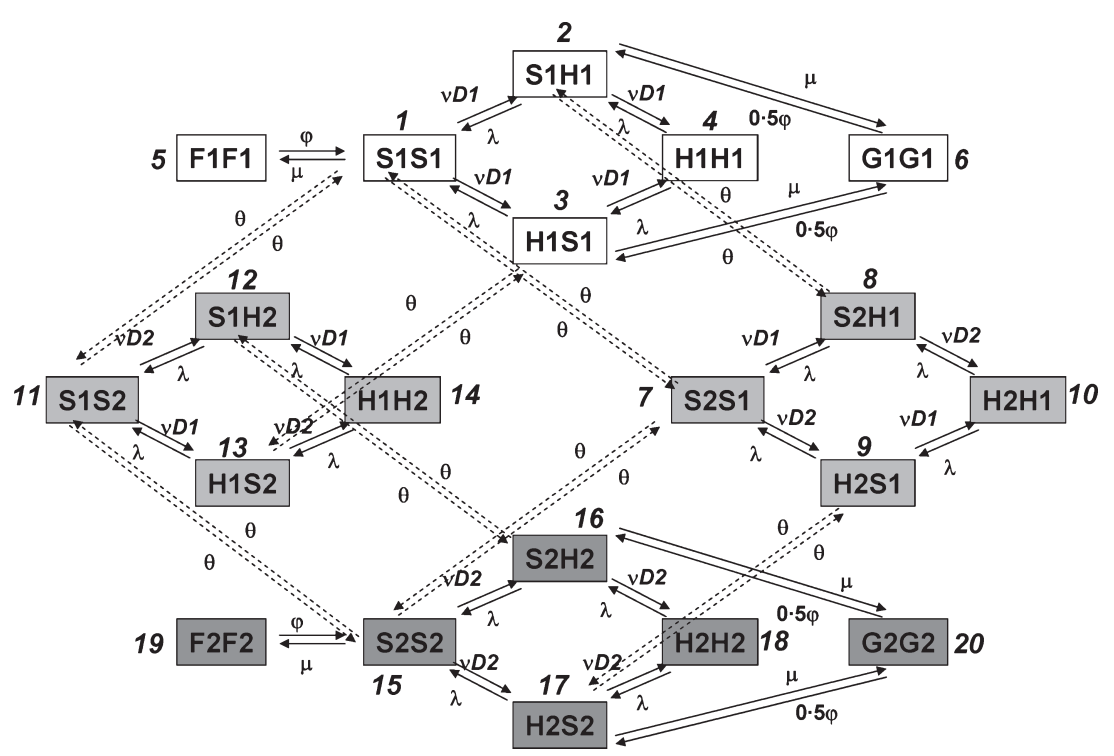

Fig. 2. A diagram of the Markov chain for two predators foraging on two patches. Each predator can be searching $(\mathrm{S})$, handling $(\mathrm{H})$, fighting after searching $(\mathrm{F})$, or fighting after handling $(\mathrm{G})$. In each of the 20 states numbers denote patch 1 or patch 2 , and the two letters denote which behaviour each predator displays. States coloured white are when both predators are on patch 1; states coloured light grey are when there is only one predator on each patch; states coloured dark grey are when both predators are on patch 2. Arrows denote transitions and transition probabilities between the states. Dashed arrows are when one predator moves from one patch to the other. 
Table 2. Balance equations and relative limiting probabilities (rlps) of the twenty states of the foraging process of two predators foraging on two patches. Rlps $\left(P_{\mathrm{i}}\right)$ are given relative to $P_{1}$, hence $P_{1}$ equals unity. The fraction search time of one predator is calculated by summing the rlps where a predator is searching on a particular patch and dividing this over the sum of all limiting probabilities

\begin{tabular}{|c|c|c|c|c|c|}
\hline & State & Rate at which leave & equals & Rate at which enter & Rlp \\
\hline 1 & $\mathrm{~S}_{1} \mathrm{~S}_{1}$ & $\left(\mu+2 v D_{1}+2 \theta\right) P_{1}$ & $=$ & $\varphi P_{1}+\lambda\left(P_{2}+P_{3}\right)+\theta\left(P_{7}+P_{11}\right)$ & $P_{1}=1$ \\
\hline 2 & $\mathrm{~S}_{1} \mathrm{H}_{1}$ & $\left(\lambda+\mu+v D_{1}+\theta\right) P_{2}$ & $=$ & $v D_{1} P_{1}+\lambda P_{4}+\frac{1}{2} \varphi P_{6}+\theta P_{8}$ & $P_{2}=v D_{1} / \lambda$ \\
\hline 3 & $\mathrm{H}_{1} \mathrm{~S}_{1}$ & $\left(\lambda+\mu+v D_{1}+\theta\right) P_{3}$ & $=$ & $v D_{1} P_{1}+\lambda P_{4}+\frac{1}{2} \varphi P_{6}+\theta P_{13}$ & $P_{3}=v D_{1} / \lambda$ \\
\hline 4 & $\mathrm{H}_{1} \mathrm{H}_{1}$ & $2 \lambda P_{4}$ & $=$ & $v D_{1}\left(P_{2}+P_{3}\right)$ & $P_{4}=\left(v D_{1} / \lambda\right)^{2}$ \\
\hline 5 & $\mathrm{~F}_{1} \mathrm{~F}_{1}$ & $\varphi P_{5}$ & $=$ & $\mu P_{1}$ & $P_{5}=\mu / \varphi$ \\
\hline 6 & $\mathrm{G}_{1} \mathrm{G}_{1}$ & $\varphi P_{6}$ & $=$ & $\mu\left(P_{2}+P_{3}\right)$ & $P_{6}=2(\mu / \varphi)\left(v D_{1} / \lambda\right)$ \\
\hline 7 & $\mathrm{~S}_{2} \mathrm{~S}_{1}$ & $\left(v D_{1}+v D_{2}+2 \theta\right) P_{7}$ & $=$ & $\theta P_{1}+\lambda\left(P_{8}+P_{9}\right)+\theta P_{15}$ & $P_{7}=1$ \\
\hline 8 & $\mathrm{~S}_{2} \mathrm{H}_{1}$ & $\left(\lambda+v D_{2}+\theta\right) P_{8}$ & $=$ & $\theta P_{2}+v D_{1} P_{7}+\lambda P_{10}$ & $P_{8}=v D_{1} / \lambda$ \\
\hline 9 & $\mathrm{H}_{2} \mathrm{~S}_{1}$ & $\left(\lambda+v D_{1}+\theta\right) P_{9}$ & $=$ & $v D_{2} P_{7}+\lambda P_{10}+\theta P_{17}$ & $P_{9}=v D_{2} / \lambda$ \\
\hline 10 & $\mathrm{H}_{2} \mathrm{H}_{1}$ & $2 \lambda P_{10}$ & $=$ & $v D_{2} P_{8}+v D_{1} P_{9}$ & $P_{10}=\left(v D_{1} / \lambda\right)\left(v D_{2} / \lambda\right)$ \\
\hline 11 & $\mathrm{~S}_{1} \mathrm{~S}_{2}$ & $\left(v D_{1}+v D_{2}+2 \theta\right) P_{11}$ & $=$ & $\theta P_{1}+\lambda\left(P_{12}+P_{13}\right)+\theta P_{15}$ & $P_{11}=1$ \\
\hline 12 & $\mathrm{~S}_{1} \mathrm{H}_{2}$ & $\left(\lambda+v D_{1}+\theta\right) P_{12}$ & $=$ & $v D_{2} P_{11}+\lambda P_{14}+\theta P_{16}$ & $P_{12}=v D_{2} / \lambda$ \\
\hline 13 & $\mathrm{H}_{1} \mathrm{~S}_{2}$ & $\left(\lambda+v D_{2}+\theta\right) P_{13}$ & $=$ & $\theta P_{3}+v D_{1} P_{11}+\lambda P_{14}$ & $P_{13}=v D_{1} / \lambda$ \\
\hline 14 & $\mathrm{H}_{1} \mathrm{H}_{2}$ & $2 \lambda P_{14}$ & $=$ & $v D_{1} P_{12}+v D_{2} P_{13}$ & $P_{14}=\left(v D_{1} / \lambda\right)\left(v D_{2} / \lambda\right)$ \\
\hline 15 & $\mathrm{~S}_{2} \mathrm{~S}_{2}$ & $\left(\mu+2 v D_{2}+2 \theta\right) P_{15}$ & $=$ & $\theta\left(P_{7}+P_{11}\right)+\varphi P_{19}+\lambda\left(P_{16}+P_{17}\right)$ & $P_{15}=1$ \\
\hline 16 & $\mathrm{~S}_{2} \mathrm{H}_{2}$ & $\left(\lambda+\mu+v D_{2}+\theta\right) P_{16}$ & $=$ & $\theta P_{12}+v D_{2} P_{15}+\lambda P_{18}+\frac{1}{2} \varphi P_{20}$ & $P_{16}=v D_{2} / \lambda$ \\
\hline 17 & $\mathrm{H}_{2} \mathrm{~S}_{2}$ & $\left(\lambda+\mu+v D_{2}+\theta\right) P_{17}$ & $=$ & $\theta P_{9}+v D_{2} P_{15}+\lambda P_{18}+\frac{1}{2} \varphi P_{20}$ & $P_{17}=v D_{2} / \lambda$ \\
\hline 18 & $\mathrm{H}_{2} \mathrm{H}_{2}$ & $2 \lambda P_{18}$ & $=$ & $v D_{2}\left(P_{16}+P_{17}\right)$ & $P_{18}=\left(v D_{2} / \lambda\right)^{2}$ \\
\hline 19 & $\mathrm{~F}_{2} \mathrm{~F}_{2}$ & $\varphi P_{19}$ & $=$ & $\mu P_{15}$ & $P_{19}=\mu / \varphi$ \\
\hline 20 & $\mathrm{G}_{2} \mathrm{G}_{2}$ & $\varphi P_{20}$ & $=$ & $\mu\left(P_{16}+P_{17}\right)$ & $P_{20}=2(\mu / \varphi)\left(v D_{2} / \lambda\right)$ \\
\hline
\end{tabular}

patch combination ' $4-16$ ' two equal crabs foraging on the rich patch would each achieve a food intake rate of 0.34 mussels per min, which for both crabs is higher than the food intake rate they would achieve alone on the poor patch ( $0 \cdot 20$ mussels per min). It turned out that feeding rewards for both crabs are predicted to always be highest on the rich patch if patches differ in quality.

Ideal-free theory yields predictions for an equilibrium situation, in which no predator can improve its feeding rate by moving to another patch. This implies that any mistake or re-sampling by the predator [referred to as non-IFD movements (Hugie \& Grand 1998)] would lead to deviations from the ideal-free hypothesis. To infer acceptance or rejection of the ideal-free hypothesis we decided that shore crabs distribute ideal and freely if they spend at least ninety percent of their foraging time on the rich patch (cf. Krebs, Kacelnik \& Taylor 1978). This has consequences for the first response variable, and entails that the expected ratio of food intakes under the ideal-free hypothesis is then given by $(0.9 \bullet$ total trial time $\left.\bullet W_{b}\right) /\left(0.1 \bullet\right.$ total trial time $\left.\bullet W_{p}\right)$, where the food intake rate $W$ is either determined by Holling's or by Beddington's equation, depending on the presence or absence of a competitor. Thus, both competitors might simultaneously spend $90 \%$ of their foraging time on the rich patch and $10 \%$ on the poor patch. Alternatively, each competitor might spend $10 \%$ of its foraging time alone on each patch and the range of the expected food intake ratio is defined by these two alternatives.

\section{Results}

When patches were of equal quality, the per capita food uptake of shore crabs did not differ between the patches, as the ratio of food intake did not differ from unity (Fig. 3a). When food patches differed in quality, shore crabs consumed more prey from the rich patch than expected under the null hypothesis of no preference when the difference in patch quality was maximal, but prey consumption did not differ from that expected under the ideal-free hypothesis (Fig. 3a). Yet, the amount of time that crabs spent on the rich patch was not different from that expected under the null hypothesis of random dispersal between patches (Fig. 3b). Furthermore, the amount of time spent on the rich patch was always lower than that expected under the ideal-free hypothesis (Fig. 3b). The time spent handling, expressed per mussel capture, was observed to be less on the rich patch than on the poor patch (Fig. 3c), because crabs adopted a so-called 'take-away strategy': crabs that were handling prey in the presence of a competitor moved to the unoccupied patch, i.e. usually the poor patch (Fig. 3d). To assess if the latter result arose because there were more opportunities to move with a prey from the rich to the poor patch, because prey density was higher on the rich patch, we expressed the number of times that a handling crab moved away from its competitor on a patch per mussel capture on that patch. Taking the difference between this proportion on the best patch and on the poor patch revealed that this differences was significantly less than zero if patches were of unequal quality (95\% bootstrap CI: $-3 \cdot 33-0 \cdot 33, n=8)$ (results for the different, unequal patch combinations were pooled because in many trials these proportions were divisions by zero). Hence handling crabs did not move more often from the rich to the poor patch, simply because the prey density was higher on the rich patch (in which case this difference would have equalled zero). This difference was not significantly different from zero when the two patches were of equal quality $(95 \%$ bootstrap CI: $-0 \cdot 22-0 \cdot 00$, $n=5$ ). It was found that the actual distribution of crabs did not reflect their attack distribution. 

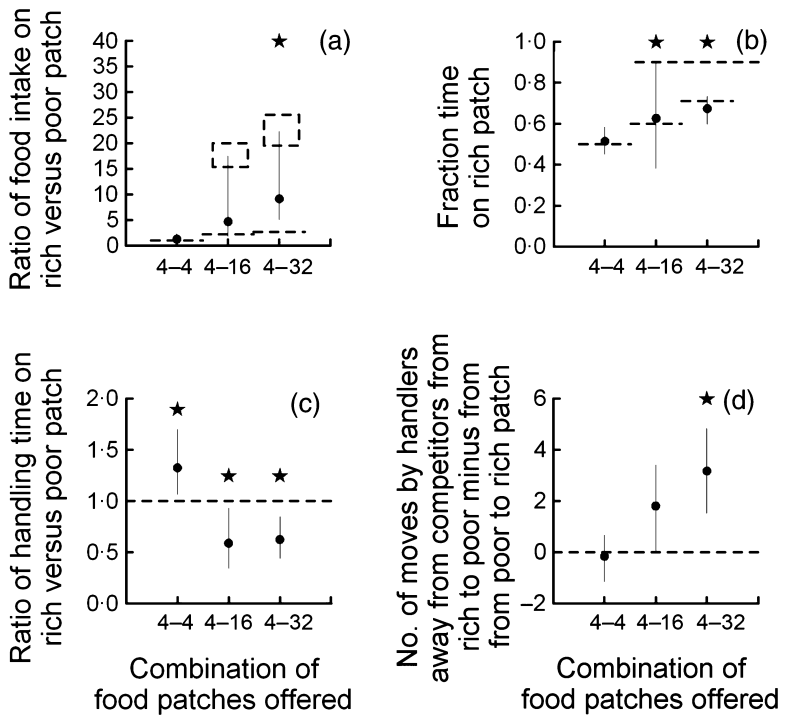

Fig. 3. Patch choice and predation of mussels by shore crabs. Numbers (e.g. 4-4) indicating the combinations of mussel patches refer to the number of mussels in a patch $\left(\right.$ area $=1 / 4 \mathrm{~m}^{2}$ ). Vertical lines are $95 \%$ confidence intervals (covered by the symbol in case of patch combination 4-4 in panel $a$ ). (a) Per capita food intake on the rich patch relative to that on the poor patch (stars denote that observations differed from the expectations under the hypothesis of equal dispersal rates [denoted by dashed lines]). Dashed boxes denote the expected range of food intake ratios under the ideal-free hypothesis (the range takes into account that crabs may forage alone or with a competitor, see Methods) (b) Average fraction of time that focal crabs spent on the rich patch (lower dashed lines denote expectations under the hypothesis of equal dispersal rates). Shore crabs are considered to distribute ideal and freely if the fraction of time spent on the rich patch was at least 0.9 (see Methods), denoted by the upper dashed line. Stars denote that observations differed from this idealfree expectation (not applicable to patch combination 4-4). (c) Handling time per mussel capture of focal crabs on the rich patch relative to that on the poor patch. Stars denote that the observed ratio differed from unity (dashed line). (d) Difference in the number of times that prey-handling focal crabs moved away from their competitor on the rich patch and away from their competitor on the poor patch. Stars denote that the observed difference was significantly different from zero (dashed line).

\section{Discussion}

We have shown that shore crabs distribute themselves homogeneously across mussel patches despite aggregating their attack where it is most profitable: their catch did not match their time spent in a prey patch. Expressed per mussel capture, crabs moved away from their competitor to the other patch more often if they were on the poor patch than if they were on the rich patch. This was likely due to the fact that crabs distributed themselves homogenously between the two patches: since crabs caught fewer prey items on the poor patch, the number of times that crabs moved to the other patch per mussel capture is then higher on the poor patch. Since crabs moved away from the poor patch more than once, even if they had only caught one mussel there, crabs likely moved back from the rich to the poor patch with their prey item, although we could not corroborate this as we had not recorded these movements.
Our results highlight an implicit assumption in the theory of ideal-free distributions: prey handling is assumed to occur in the patch where the prey was caught. Thus, it is important to distinguish between the sites where predators catch their prey and where they perform other activities (e.g. consumption). This distinction is also important because the attack distribution of predators has been shown to be decisive for persistence and stability of predator and prey in ecological models (Hassell \& May 1973; May 1978; Murdoch \& StewartOaten 1989; Durrett \& Levin 1994). Thus, contrary to the vast majority of empirical studies (Stiling 1987; Tregenza 1995; MacLean et al. 2005), spatial patterns of forager abundance should not be taken as the distributions of prey consumption. Our result on the mismatch between prey catch and time spent in the prey patch is robust against variation in our predicted ideal-free distribution where $90 \%$ of all foraging time should be spent on the rich patch. First, increasing this percentage would not change the fact that crabs spent significantly less time on the rich patch than predicted under the ideal-free hypothesis (see Fig. 3b). Secondly, for crabs that aggregated their attack on the most profitable patch (patch combination '4-32'), decreasing this percentage would affect the results only if the ideal-free percentage would fall within the $95 \%$ confidence interval around the mean percentage of time that crabs spent on the rich patch. For patch combination '4-32' this means that the ideal-free prediction has to be reduced to such an extent that it would be similar to the prediction under the null hypothesis (Fig. 3b), which is biologically unrealistic.

One could argue that the movement of crabs away from the rich patch towards the poor patch is an artefact because the two patches were relatively small and adjacent to one another. However, in an underwater field study where an artificial patch of mussels was created (which was four times smaller $\left(0.06 \mathrm{~m}^{2}\right)$ than each food patch in our study) it was frequently observed that if the patch was occupied by a shore crab, other shore crabs did not stay on the patch but made brief visits to the patch to try removing mussels from the clump (Cunningham 1983). If a crab was successful it did not stay on the patch with the other crab, but carried the mussel away to consume it (Cunningham 1983). Crabs regularly revisited the patch, sometimes up to eight times within the three hours of observation (Cunningham 1983). From these observations we infer that the movement patterns of the crabs in our experiments were not an artefact of the experimental setup, but a result of adopting 'take-away' strategies that crabs may adopt in the wild.

This prompts the question as to why predators would decide to leave a high-quality food patch after attack on a prey. In our study, crabs could reduce interference such as kleptoparasitism by moving their victim to the other patch that has no competitor. This phenomenon is probably not unique for crabs that forage on mussels. It might be general to predator-prey systems where handling times of prey are long and foragers can move freely with prey. For instance, oystercatchers Haematopus ostralegus are known to avoid each other while foraging (Vines 1980) and, as competitor density increases, oystercatchers carry prey away from where 
they were found prior to attempting to open them (Norris \& Johnstone 1998). Also, hermit crabs Pagurus pollicaris have been observed to tear off pieces from fish bait to consume it away from the aggregation of crabs around the bait (Hazlett 1979). Furthermore, in a field experiment it was observed that the rock crab Cancer irroratus aggregated its prey attack on patches where the density of sea scallops Placopecten magellanicus was high, but its actual distribution was uncorrelated with patch quality (Barbeau, Scheibling \& Hatcher 1998)

A reason different from avoiding kleptoparasitism might be that crabs attempt to avoid being killed by a cannibalistic competitor (Moksnes, Pihl \& van Montfrans 1998). This was suggested by Sih (1980) to explain why juvenile backswimmers at risk of cannibalism distributed equally between two food patches of unequal quality. Sih (1980) measured feeding rates of backswimmers to derive ideal-free predictions on patch choice. Both juvenile and adult backswimmers had a choice between a high-quality food patch and a low-quality patch. The results showed that both were able to assess patch quality. Adult backswimmers were present at the high-quality food patch most of the time. Since juveniles were at risk of cannibalism, they spent only $50 \%$ of their time on the rich patch, despite the fact that they could achieve the highest feeding rate at the high-quality patch. Interestingly, juvenile backswimmers did not experience a reduction in feeding rate (Sih 1982). The results therefore suggest that, in the absence of significant travelling costs, juveniles can reduce their risk of predation by distributing evenly between patches (Sih 1980), while still being able to achieve maximum feeding rates in the rich patch.

During the experiments, crabs still engaged in agonistic interactions, which might have served to either assess a potential prey or to ward off a potential predator, as food patches were small enough to bring crabs always within the sensory range of a competitor (Warner 1977). This avoidance response is observed for juvenile Dungeness crabs Cancer magister where in various field experiments crabs consistently dispersed from initially high densities, irrespective of food availability (Iribarne, Fernandez \& Armstrong 1994). This avoidance response is also observed in other species where individuals are susceptible to cannibalism. In laboratory situations, antlion larvae Myrmeleon acer and larvae of the cotton bollworm Helicoverpa armigera were initially distributed in a clumped fashion within a patch, but as more conspecifics were introduced, animals distributed increasingly uniformly (Day \& Zalucki 2000; Kakimoto, Fujisaki \& Miyatake 2003). A final reason why animals avoid conspecifics might be that their predators exert pressure on animals to space out (Tinbergen, Impekoven \& Franck 1967). Such spacing-out behaviour to avoid predation is commonly observed in species whose main defence is to avoid detection, and where the distance at which a predator detects them is larger than the distances between individuals (Kennedy \& Crawley 1967; Andersson \& Wiklund 1978).

If the implicit assumption of IFD theory is not met, as is the case for at least several species exhibiting mutual inter- ference and avoidance, IFD models only predict the preyattack distribution of predators. Whether or not it matters if the attack distribution differs from the actual distribution of predators will foremost depend on the type of predatorprey model used. For example, in the classic Lotka-Volterra model, heterogeneity in the attack distribution confers stability to predator-prey systems (Diekmann et al. 2005). If, however, the observed, homogeneous distribution of predators is taken as the attack distribution, the predatorprey dynamics are, incorrectly, predicted to be unstable. In other types of predator-prey models, the consequences of not meeting the implicit assumption of IFD theory may depend on the degree of aggregation in prey attack that is required for stable predator-prey dynamics (Hassell \& May 1973; May 1978; Bernstein, Auger \& Poggiale 1999). In order to predict and understand the actual distribution of predators, one should consider how predators balance their need to maximize food intake rate and their need to reduce the risk of interference and predation. As yet, IFD models with kleptoparasitism (Korona 1989; Holmgren 1995; Ruxton \& Moody 1997; Hamilton 2002) have not incorporated the movement of handlers. If only handlers move between patches (e.g. because they can actively keep a distance from their competitor), model predictions are expected to change, but these have not yet been derived (Hamilton 2002). If kleptoparasites are allowed to move between patches as well, decisions to kleptoparasitize will depend on the costs and benefits of kleptoparasitism and those of foraging for food in the conventional way. To what extent the resulting model predictions change remains to be derived.

Predator-prey systems where predators are at risk of being cannibalized are comparable to systems characterized by intraguild predation where predators (or the stronger individuals of a species) and prey (or the weaker individuals of the same species) feed on a basal resource, yet also eat prey. However, the only IFD model that incorporates intraguild predation (Heithaus 2001) does not take interference competition between predators or between preys into account. Empirical results (Sih 1980; Day \& Zalucki 2000; Kakimoto, Fujisaki \& Miyatake 2003; this study) suggest that animals at risk of kleptoparasitism or predation tend to distribute equally between patches.

\section{Acknowledgements}

The authors would like to thank Wouter Vahl for discussion and comments, and the Netherlands Organization for Scientific Research (NWO) for funding (research grant and Rubicon Fellowship). Experiments conformed to Dutch law on the use of laboratory experiments.

\section{References}

Andersson, M. \& Wiklund, C.G. (1978) Clumping versus spacing out: experiments on nest predation in fieldfares (Turdus pilaris). Animal Behaviour, 26, 1207-1212.

Barbeau, M.A., Scheibling, R.E. \& Hatcher, B.G. (1998) Behavioural responses of predatory crabs and sea stars to varying density of juvenile sea scallops. Aquaculture, 169, 87-98. 
Beddington, J.R. (1975) Mutual interference between parasites and predators and its effect on searching efficiency. Joural of Animal Ecology, 44, 331-340.

Bernstein, C., Auger, P. \& Poggiale, J.C. (1999) Predator migration decisions, the ideal free distribution, and predator-prey dynamics. American Naturalist, 153, 267-281.

Chesson, P.L. \& Murdoch, W.W. (1986) Aggregation of risk: relationships among host-parasitoid models. American Naturalist, 127, 696-715.

Cunningham, P.N. (1983) Predatory activities of shore crab populations. $\mathrm{PhD}$ thesis, University of Wales, Cardiff.

Day, M.D. \& Zalucki, M.P. (2000) Effect of density on spatial distribution, pit formation and pit diameter of Myrmeleon acer Walker, (Neuroptera: Myrmeleontidae): patterns and processes. Austral Ecology, 25, $58-64$.

Diekmann, O., Cheng-fu, J., Metz, J.A.J. \& Sabelis, M.W. (2005). Appendix differential prey patch vulnerability stabilizes predator-prey interaction. In M.W. Sabelis, A. Janssen, O. Diekmann, V.A.A. Jansen, E. Van Gool \& M. Van Baalen. Global persistence despite local extinction in acarine predatorprey systems: Lessons from experimental and mathematical exercises. Advances in Ecological Research, 37, 183-220.

Durrett, R. \& Levin, S.A. (1994) The importance of being discrete (and spatial). Theoretical Population Biology, 46, 363-394.

Fretwell, S.D. \& Lucas, H.L. (1969) On territorial behaviour and other factors influencing habitat distribution in birds. Acta Biotheorica, 19, 16-36.

Hamilton, I.M. (2002) Kleptoparasitism and the distribution of unequal competitors. Behavioral Ecology, 13, 260-267.

Hassell, M.P. (2000) The Spatial and Temporal Dynamics of Host-parasitoid Interactions. Oxford University Press, Oxford.

Hassell, M.P. \& May, R.M. (1973) Stability in insect-host parasite systems. Journal of Animal Ecology, 42, 693-736.

Hazlett, B.A. (1979) Individual distances in crustacea: IV. Distance and dominance hierarchies in Pagurus pollicaris. Marine Behavior \& Physiology, 6 225-242.

Heithaus, M.R. (2001) Habitat selection by predators and prey in communities with asymmetrical intraguild predation. Oikos, 92, 542-554.

Holling, C.S. (1959) Some characteristics of simple types of predation and parasitism. Canadian Entomologist, 91, 385-398.

Holmgren, N. (1995) The ideal free distribution of unequal competitors: predictions from a behaviour-based functional response. Journal of Animal Ecology, 64, 197-212.

Hugie, D.M. \& Grand, T.C. (1998) Movement between patches, unequal competitors and the ideal free distribution. Evolutionary Ecology, 12, 1-9.

Iribarne, O., Fernandez, M. \& Armstrong, D. (1994) Does space competition regulate density of juvenile Dungeness crab Cancer magister Dana in sheltered habitats? Journal of Experimental Marine Biology and Ecology, 183, 259-271.

Kakimoto, T., Fujisaki, K. \& Miyatake, T. (2003) Egg laying preference, larval dispersion, and cannibalism in Helicoverpa armigera (Lepidoptera : Noctuidae). Annals of the Entomological Society of America, 96, 793-798.

Kennedy, J.S. \& Crawley, L. (1967) Spaced-out gregariousness in sycamore aphids Drepanosiphum platanoides (Schrank) (Hemiptera, Callaphididae) with a statistical appendix by A.D. McLaren. Journal of Animal Ecology, 36, 147-170.

Korona, R. (1989) Ideal free distribution of unequal competitors can be determined by the form of competition. Journal of Theoretical Biology, 138, 347352.

Krebs, J.R., Kacelnik, A. \& Taylor, P. (1978) Test of optimal sampling by foraging great tits. Nature, 275, 27-31.

Lotka, A.J. (1925) Elements of Physical Biology. Williams and Wilkins, Baltimore.

MacLean, A., Huntingford, F.A., Ruxton, G.D., Morgan, I.J., Hamilton, J. \& Armstrong, J.D. (2005) Testing the assumptions of the ideal despotic distribution with an unpredictable food supply: experiments in juvenile salmon. Journal of Animal Ecology, 74, 214-225.

May, R.M. (1978) Host-parasitoid systems in patchy environments: a phenomenological model. Journal of Animal Ecology, 47, 833-841.

van der Meer, J. \& Smallegange, I.M. (2009) A stochastic version of the Beddington-DeAngelis functional response: modelling interference for a finite number of predators. Journal of Animal Ecology, 78, 134-142.

Milinski, M. \& Parker, G.A. (1991) Competition for resources. An Introduction to Behavioural Ecology (eds J.R. Krebs \& N.B. Davies), pp. 102-119, Blackwell Scientific Publications, Oxford.

Moksnes, P.O., Pihl, L. \& van Montfrans, J. (1998) Predation on postlarvae and juveniles of the shore crab Carcinus maenas: importance of shelter, size and cannibalism. Marine Ecology Progress Series, 166, 211225 .
Murdoch, W.W. \& Stewart-Oaten, A. (1989) Aggregation by parasitoids and predators: effects on equilibrium and stability. American Naturalist, 134, 288-310.

Nicholson, A.J. \& Bailey, V.A. (1935) The balance of animal populations. Part 1. Proceedings of the Zoological Society of London, 3, 551-598.

Norris, K. \& Johnstone, I. (1998) Interference competition and the functional response of oystercatchers searching for cockles by touch. Animal Behaviour, 56, 639-650.

Ranta, E., Lundberg, P. \& Kaitala, V. (2006) Ecology of Populations. Cambridge University Press, Cambridge.

Rosenzweig, M.L. \& MacArthur, R.H. (1963) Graphical representation and stability conditions of predator-prey interactions. American Naturalist, 97, 209-223.

Ross, S.M. (1989) Introduction to Probability Models. Academic Press, Inc, San Diego.

Ruxton, G.D. \& Moody, A.L. (1997) The ideal free distribution with kleptoparasitism. Journal of Theoretical Biology, 186, 449-458.

Sih, A. (1980) Optimal behavior: can foragers balance two conflicting demands? Science, 210, 1041-1043.

Sih, A. (1982) Foraging strategies and the avoidance of predation by an aquatic insect, Notonecta hoffmanni. Ecology, 63, 786-796.

Smallegange, I.M. \& van der Meer, J. (2003) Why do shore crabs not prefer the most profitable mussels? Journal of Animal Ecology, 72, 599-607.

Smallegange, I.M. \& van der Meer, J. (2007) Interference from a game theoretical perspective: shore crabs suffer most from equal competitors. Behavioral Ecology, 18, 215-221.

Smallegange, I.M. \& van der Meer, J. (2010) Testing a stochastic version of the Beddington-DeAngelis functional response in foraging shore crabs. Marine Biology, 157, 1027-1040

Smallegange, I.M., van der Meer, J. \& Kurvers, R.H.J.M. (2006) Disentangling interference competition from exploitative competition in a crab-bivalve system using a novel experimental approach. Oikos, 113, 157-167.

Stiling, P.D. (1987) The frequency of density dependence in insect host-parasitoid systems. Ecology, 68, 844-856.

Tinbergen, N., Impekoven, M. \& Franck, D. (1967) An experiment on spacingout as a defence against predation. Behaviour, 28, 307-321.

Tregenza, T. (1995) Building on the ideal free distribution. Advances in Ecological Research, 26, 253-307.

Van Baalen, M. \& Sabelis, M.W. (1993) Coevolution of patch selection strategies of predator and prey and the consequences for ecological stability. American Naturalist, 142, 646-670.

Van Baalen, M. \& Sabelis, M.W. (1999) Non-equilibrium population dynamics of 'ideal and free' prey and predators. American Naturalist, 154, 69-88.

Vines, G. (1980) Spatial consequences of aggressive-behavior in flocks of oystercatchers, Haematopus-ostralegus L. Animal Behaviour, 28, 1175-1183.

Volterra, V. (1926) Fluctuations in the abundance of a species considered mathematically. Nature, 118, 558-600.

Walde, S. \& Murdoch, M.M. (1988) Spatial density dependence in parasitoids. Annual Review of Entomology, 33, 441-466.

Warner, G.F. (1977) The Biology of Crabs. Paul Elek (Scientific Books) Ltd, London.

Received 17 December 2009; accepted 29 March 2010

Handling Editor: Tim Coulson

\section{Supporting Information}

Additional Supporting Information may be found in the online version of this article.

Fig. S1. Stochastic runs of the 2-patch-2-predator Markov chain, showing that the fraction of time that two individuals spend searching, handling or fighting on each patch, converges to a limiting value.

Table S1. Balance equations of the six states of the foraging process of one or two predators foraging in one patch.

As a service to our authors and readers, this journal provides supporting information supplied by the authors. Such materials may be re-organized for online delivery, but are not copy-edited or typeset. Technical support issues arising from supporting information (other than missing files) should be addressed to the authors. 